\title{
Kernos
}

Revue internationale et pluridisciplinaire de religion grecque antique

$14 \mid 2001$

Varia

\section{Le Rhésos et l'orphisme}

\section{Caroline Plichon}

Édition électronique

DOI : 10.4000/kernos.762

ISSN : 2034-7871

\section{Éditeur}

Centre international d'étude de la religion grecque antique

\section{Édition imprimée}

Date de publication : 1 janvier 2001

Pagination : 11-21

ISSN : 0776-3824

Référence électronique

Caroline Plichon, « Le Rhésos et l'orphisme », Kernos [En ligne], 14 | 2001, mis en ligne le 14 avril 2011 consulté le 01 mai 2019. URL : http://journals.openedition.org/kernos/762 ; DOI : 10.4000/kernos.762 
Kernos, 14 (2001), p. 11-21.

\section{Le Rbésos et l'orphisme*}

Le Rhésos s'achève sur une scène de deus ex machina. Rhésos, le roi thrace qui donne son nom à la tragédie, s'est fait tuer par Ulysse et Diomède sur les indications d'Athéna. C'est sa mère, une Muse, qui intervient à la fin de la pièce ( $v$. 890-982). Après s'être présentée (v. 890-894), elle se lamente dans une monodie (v. 895-903 et v. 906-914). Sa lamentation est aussi cri de vengeance, contre Ulysse et Diomède, contre Thamyris ${ }^{1}$, et surtout contre Athéna (v. 938-949). La Muse accuse l'ingratitude d'Athéna envers les Muses qui ont honoré Athènes de leurs bienfaits : non seulement les Muses avaient fait d'Athènes leur terre d'élection mais, de plus, leur parent Orphée, fils d'une Muse et cousin de Rhésos (v. 943-944), y a introduit les mystères. Nous trouvons une seconde mention d'Orphée ( $v$. 966), liée à la première, dans ce que dit la Muse, à partir du vers 962, sur le sort qui attend Rhésos après sa mort. En effet, elle affirme qu'elle va demander à Perséphone de relâcher l'âme de Rhésos des Enfers, ce que Perséphone est tenue de faire parce qu'elle lui doit d'honorer ceux qui sont chers à Orphée. (v. 965-966). Ainsi, la Muse dessine un réseau de liens entre des figures (Orphée, Déméter, Perséphone) qui appartiennent toutes à la sphère mystique, et affilie l'orphisme et les mystères éleusiniens. Enfin, une autre divinité appartenant à la sphère mystique, Bacchos, apparaît plus loin dans son discours, lorsqu'elle dit ce que deviendra Rhésos une fois relâché des Enfers (v. 972).

Dans ce passage du Rhésos sont donc rassemblés des éléments qui ont tous partie liée avec la question, obscure et complexe, de l'« orphisme ${ }^{2}$,

- Cet article est le texte remanié d'une communication présentée d'abord dans le séminaire de $\mathrm{Ph}$. Rousseau à l'université de Lille III, puis dans le cadre du $\mathrm{XI}^{\mathrm{e}}$ colloque «CorHaLi » (Cornell, Harvard, Lille), organisé et dirigé par C. Calame, qui s'est tenu à Lausanne en mai 2000 sur le thème de «La figure d'Orphée et les poèmes orphiques ». Je tiens également à remercier P. Judet de La Combe et F. Blaise pour leurs conseils. J'ai pu bénéficier d'une allocation de recherche financée par la région Nord-Pas de Calais.

1 Thamyris est l'aède thrace qui avait défié les Muses dans un concours de chant ( $c f$. Iliade II, 594-690). C'est en se rendant à ce concours, qui a lieu - chez Euripide - sur le mont Pangée, que la Muse franchit le Strymon qui la viole.

2 J'emploie ce terme dans son acception élargie (à la manière de D. SABBATucci dans son Essai sur le mysticisme grec, Paris, 1982 (traduction par J.P. Darmont de Saggio sul 
éléments dont les relations réciproques posent problème aux historiens des religions. $\mathrm{Si}$, en présence de ces éléments, la tentation est grande de donner une interprétation " orphique " au passage, on se rend compte, en examinant le texte, que celle-ci se heurte à des incohérences et ne peut être menée à terme. Le texte poétique est avant tout à considérer, non comme un témoignage mais comme une construction, dont il nous faut comprendre la logique propre. Les éléments orphiques n'en sont qu'un matériau que le poète utilise comme il le fait, ainsi que nous le verrons, de la matière hésiodique. Une approche philologique, qui tente de reconstituer l'univers propre du texte, nous fera entrevoir qu'il s'agit d'un univers hétérogène et que le poète du Rbésos, loin d'affirmer une croyance, construit une énigme.

Examinons donc dans un premier temps les éléments qui ont fait de ce passage du Rhésos une pièce du dossier concernant l'orphisme et voyons quelles sont les limites d'une interprétation orphique dans ce cadre.

La Muse dit aux vers 943-944 qu'Orphée a fait connaître à Athènes « les

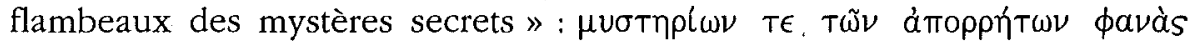

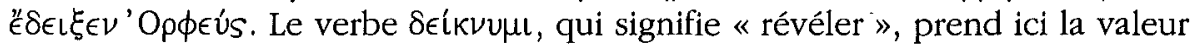

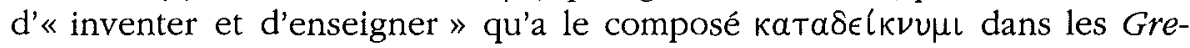
nouilles d'Aristophane (v. $1030 \mathrm{sq}$ ). Il y est dit à propos d'Orphée :'O $\rho \phi \in u ́ s$

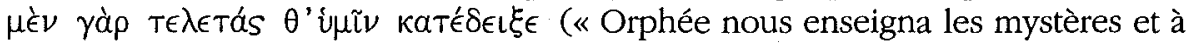
nous abstenir des meurtres "). L'adjectif ámoppńtos est interchangeable, dans

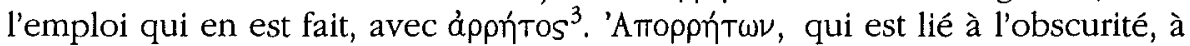
ce qui est caché, s'oppose dans le vers à фavás qui implique au contraire la lumière, la clarté : si l'on peut dire que $\mu v \sigma \pi \eta \rho ́ \omega \nu$ фavás est utilisé par synec-

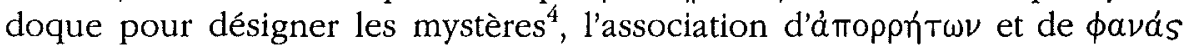
montre que, d'après l'enseignement d'Orphée, il y a de la lumière dans ce qui est caché et ne peut être dit. La révélation des mystères est une illumination. Elle éclaire, elle fait sortir de la nuit. Or, c'est aussi ce que fait la parole de la Muse en cette fin de tragédie : la Muse offre aux Troyens de voir ce qui leur a

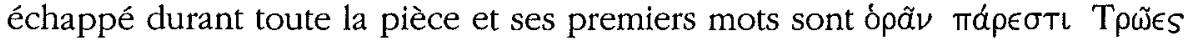
(v. 890). Le poète du Rhésos joue avec la topique traditionnelle de la Muse, en la détournant. En effet, dans l'épopée, la Muse vient chanter par l'intermédiaire de l'aède ce qui n'est pas sous les yeux de ceux qui l'écoutent: l'auditoire ne peut voir les batailles se dérouler devant lui, il doit les imaginer. Elle-même appartient au domaine de l'invisible; elle n'est pas là lorsque chante l'aède, elle n'est que la source de son discours. De même, chez Hésiode, les Muses sont nocturnes et enveloppées de brume (Théogonie, 9-

misticismo greco, Rome, 1965, p. 45) puisque j'examinerai la possibilité d'une interprétation plus particulièrement pythagoricienne.

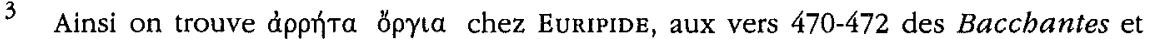

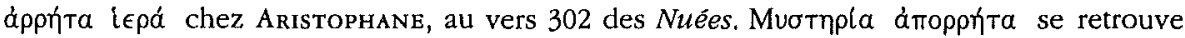
quant à lui au vers 442 de l'Assemblée des femmes.

4 Cf. I.M. LINFonth, The Arts of Orpheus, New York, 1973, p. 63. 
10). Ainsi, le fait qu'une Muse dise qu'il est permis de voir est inattendu, contraire à l'essence de la Muse homérique ou hésiodique. Celle du Rbésos est visible et le chour a le droit de la regarder : elle insiste sur ce fait par le

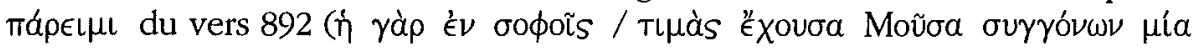
/ $\pi a ́ \rho \in(\mu \iota . .$.$) : elle est présente, et ce n'est pas exclusivement aux côtés de$ l'aède, comme dans l'épopée, mais aux côté du commun des mortels, les Troyens. Le poète du Rbésos fait ici référence au vers 485 de l'Iliade : úfeĩs

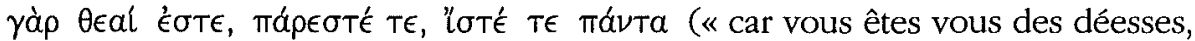
vous êtes présentes, vous savez tout »), où le verbe $\pi a ́ \rho \epsilon \iota \mu$ a posé des problèmes d'interprétation. On s'est demandé en effet si cela signifiait que les Muses sont présentes aux côtés de l'aède pour l'assister (ce qui est sans doute un sens du verbe ici), ou si cela signifiait qu'elles sont présentes aux événements. À cet endroit de l'épopée, l'aède a particulièrement besoin des Muses pour l'assister dans le catalogue des vaisseaux car elles seules savent qui étaient les chefs des Danaens. Quand il dit qu'elles sont présentes

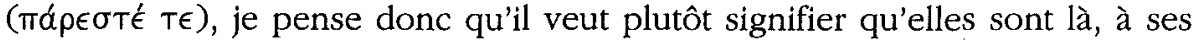
côtés, prêtes à l'aider à ce moment particulier. Dans le Rbésos, il semble y avoir rencontre entre les deux sens possibles du verbe $\pi a ́ p \in \iota \mu$ car la Muse est à la fois présente au chant et présente à l'événement, les deux moments coïncidant dans la tragédie. Le poète fait jouer pleinement la double significa-

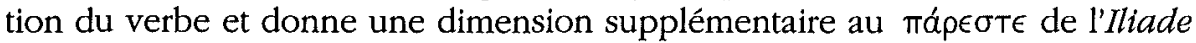
en introduisant une Muse dans l'action, sur le champ de bataille, ce qui n'est pas dans l'épopée, où les Muses sont des témoins extérieurs à l'action. Dans notre passage, le poète du Rbésos poursuit son travail de réécriture. Ainsi, il est curieux que les Muses soient associées aux mystères, elles qui divulguent une poésie ouverte à tous ${ }^{5}$. À travers cette association des Muses et des mystères, il y a en quelque sorte effacement des distinctions : le culte est subsumé sous la poésie. La Muse et Orphée ont tous deux en commun le même pouvoir d'illumination.

On s'est demandé ce qu'étaient ces mystères révélés par Orphée : certains y voient une référence aux mystères en général (C.A. Lobeck) ${ }^{6}$, pour d'autres il s'agit des mystères d'Agra (E. Maass) ${ }^{7}$, pour d'autres des mystères d'Éleusis (O. Gruppe) $)^{8}$. I.M. Linforth ${ }^{9}$ ne se prononce pas, mais insiste sur le fait que

5 Cf. M. Detienne, Homère, Hésiode et Pytbagore. Poésie et philosopbie dans le pythagorisme ancien, Bruxelles, 1962, p. 15 : «Lorsque les mystères firent en Grèce leur apparition dans la vie littéraire, un conflit s'éleva; il opposait ce que nous appellerions une poésie laïque et civique, celle d'Homère et d'Hésiode, à la poésie religieuse et sacerdotale que l'Antiquité attribue à Orphée et Musée. »

6 C.A. Lовеск, Aglaophamus sive de theologiae mysticae Graecorum causis, Leipzig, 1829, I, p. 239.

7 E. Masss, Orpheus, Munich, 1895, p. $72 s q$.

8 O. GrupPe, "Orpheus", in W.H. Roscher, Ausfürbliches Lexicon der griechischen und römiscben Mythologie, Leipzig, 1897-1901, p. 1096.

9 O.c. (n. 4), p. 64 
ces mystères, d'après ce que dit la Muse, étaient honorés à Athènes et sont présentés par elle comme une faveur accordée à cette cité, sans que soient mentionnées les autres. Les rapports entre Éleusis et l'orphisme ne nous sont pas bien connus et c'est pourquoi ces vers du Rhésos ont été discutés. Orphée est présenté comme fondateur d'Éleusis dans des vers postérieurs à 300 ap. J.-C et, pour ce qui est de la période antérieure, chez Aristophane (Grenouilles, v. $1030 \mathrm{fiq}$ ) comme chez le pseudo-Démosthène (XXV, 11), les télétai qu'a introduits Orphée ne sont pas spécifiés ${ }^{10}$. Il est vrai que, dans ces vers du Rbésos, nous sommes incités à voir une allusion aux mystères d'Éleusis qui, pour les Athéniens, comme le souligne W. Burkert, étaient les mystères «tout court ${ }^{11}$. Outre le vocabulaire employé, les vers $965-966$ permettent également d'y voir une référence à Éleusis : la Muse y dit que

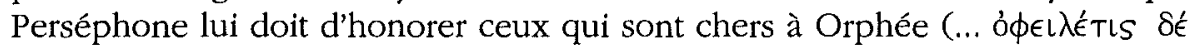

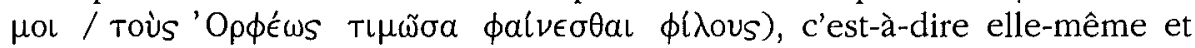
Rhésos, le cousin d'Orphée. Si elle doit cela, nous pouvons supposer que c'est parce qu'Orphée a introduit les mystères de Déméter et Perséphone à Athènes. La Muse a donc établi un lien entre l'orphisme et les mystères éleusiniens, mais aussi entre le culte et la poésie puisqu'Orphée est présenté avant tout comme un envoyé des Muses, lui-même fils d'une Muse.

Si ce passage du Rbésos offre matière à une interprétation « orphique ", c'est aussi parce que la Muse y annonce pour son fils un avenir post mortem privilégié, qui présente des éléments renvoyant à une eschatologie. Celle-ci est néanmoins impossible à reconstituer. En effet, la Muse demande à Perséphone de «faire remonter » l'âme de Rhésos des Enfers (v. 965 : \&uxìv

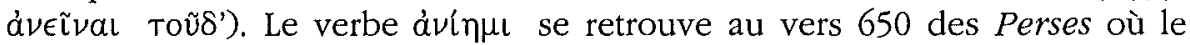
chœur demande à Hadès de faire remonter au jour l'âme de Darius. Il est proche du verbe $\alpha \nu \alpha \delta i \delta \omega \mu$ que l'on retrouve cette fois dans un passage du Ménon de Platon (81b-81e) sur l'immortalité de l'âme, où l'on a vu l'exposé d'une doctrine ou orphique ou pythagoricienne : il y est dit que Perséphone renvoie $(\dot{\alpha} \nu \delta เ \delta o \tilde{l})$ de nouveau l'âme de certains hommes. La destinée de Rhésos, après que Perséphone a relâché son âme, n'est cependant pas la même que celle des hommes du Ménon: Rhésos, lui, gît sous le sol en tant

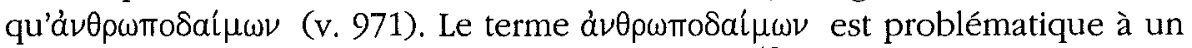
double titre: d'abord parce que c'est un bapax ${ }^{12}$ pour l'époque classique, ensuite parce que l'un des termes du composé, $\delta a i ́ \mu \omega \nu$, pose lui-même problème. Comme le dit $M$. Detienne ${ }^{13}$, « $\delta a l \mu \omega \nu$ est un signifiant dont le signifié

10 Pour F. GraF (Eleusis und die orpbiscbe Dicbtung Atbens in vorbellenistiscber Zeit, Berlin/New York, 1974, p. 22-39), chez Aristophane et chez le ps.-Démosthène, comme dans le Rbésos, le vocabulaire employé indique qu'il s'agit des mystères éleusiniens, $C f$. également Linforth, o.c. (n. 4), p. 189-197.

11 W. Burkert, Ancient Mystery Cults, Cambridge, 1987, p. 4.

12 Ce nom composé ne réapparaît que chez Procope, historien du vi ${ }^{\mathrm{e}}$ siècle ap. J.-C.

13 M. Detinnne, De la pensée religieuse à la pensée pbilosopbique. La notion de daimôn dans le pythagorisme ancien, Paris, 1963, p. 27. 
n'a pas une valeur fixe et déterminée dans la pensée religieuse. » Ainsi, $\delta a i ́ \mu \omega \nu$ à l'intérieur d'une seule pièce comme le Rhésos, peut avoir plusieurs significations différentes déterminées par le contexte ${ }^{14}$. Ici, il est employé pour désigner ce que devient un homme après sa mort. Cette signification de $\delta a l \mu \omega \nu$ trouve son origine chez Hésiode (Trav., 123 sq); nous la retrouvons, dans la tragédie, aux vers 620 et 641 des Perses où Darios est appelé $\delta a i \mu \omega \nu$,

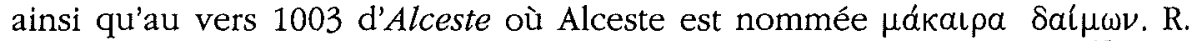
Schlesier note, dans une étude sur la notion de $\delta a i \mu \omega \nu$ chez Euripide ${ }^{15}$, que cette signification est marginale dans l'œuvre du dramaturge. En revanche, elle a été annexée et transformée par la pensée philosophique. Ainsi le $\delta a i ́ \mu \omega \nu$ apparaît-il chez les Pythagoriciens ${ }^{16}$, chez Empédocle, et plus tard, chez Platon. La proximité de l'orphisme et du pythagorisme, ainsi que le contexte mystique de notre passage, nous amènent à nous demander si une interprétation pythagoricienne du daimôn du vers 971 est possible et si c'est une eschatologie de type mystique que le poète du Rhésos nous expose ici. Cette hypothèse ne peut être envisagée bien longtemps car nous ne trouvons rien dans le texte pour la confirmer. S'il est bien question pour les daimones

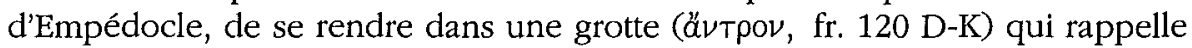
celles où sera caché Rhésos (ăvTposs, v. 970), il ne s'agit pour eux que d'un

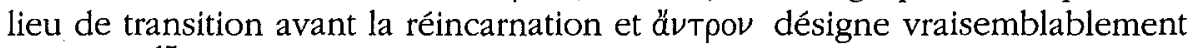
les Enfers ${ }^{17}$. Pour Rhésos au contraire, ce séjour est définitif et il ne s'agit pas des Enfers puisque Perséphone en a fait remonter son âme. Il n'est pas non plus question pour lui de réincarnation. La description de sa vie post mortem ne ressemble pas à celle que nous trouvons dans les discours mystiques. Ainsi, il est seul : il ne rejoint pas d'autres bienheureux. Enfin, ce n'est pas en raison de sa vertu qu'il bénéficie de ce statut privilégié, mais parce que Perséphone est liée à la Muse par une dette, ce qui est d'ailleurs l'inverse de ce que l'on attendrait, à savoir que ce soit le défunt qui ait à s'acquitter de la sorte.

La demeure souterraine, qui sera celle de Rhésos après la mort, est une caractéristique des daimones hésiodiques, qui ont été repensés par les

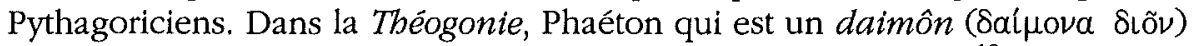

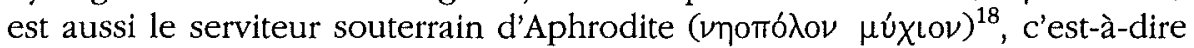

14 Au vers 56 du Rbésos, Hector s'adresse à la divinité qui préside aux événements et

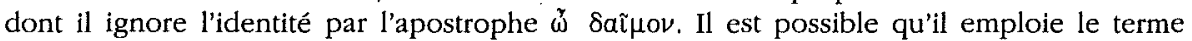
au sens de "chance ", "destin ", comme le prouve dans la suite le verbe $\epsilon u ̛ r u \chi \epsilon ́ \omega$.

15 R. Schlesier, "Daimon und Daimones bei Euripides", Saeculum 34 (1983), p. 267 279, voir plus particulièrement p. 276. Cette étude ne prend pas en compte le Rbésos.

16 Voir l'étude de Detienne, o.c. (n. 13).

17 Cf. A. Martin, O. Primavesi, L'Empédocle de Strasbourg, Berlin/New York, De Gruyter, 1999, p. 316.

$18 \mu u ́ x$ เov est une leçon d'Aristarque, alors que les manuscrits donnent vúxเov. Nous pensons avec M.L. WEst (Hesiod Theogony, Oxford, 1966, ad loc.) que $\mu$ úxtov est la leçon appropriée au contexte. 
qu'il a sa demeure sous la terre. G. Nagy ${ }^{19}$ affirme avec E. Rodhe ${ }^{20}$ qu'en dépit de l'épithète émı $\chi \forall$ óvıo qui les caractérisé, les daimones de la race d'or (Trav, 123 sq.) ont probablement aussi leur demeure sous la terre, tout comme les hommes de la race d'argent. La qualification d'ả $\nu \rho \omega \pi 0 \delta a i \mu \omega \nu$, qui unit la mortalité à la divinité, différencie Rhésos de Phaéton qui, lui, est $\delta a\lfloor$ iova $\delta \iota \tilde{\nu}$, et le rapproche en revanche des hommes de la race d'argent

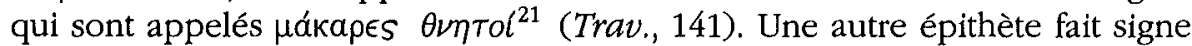

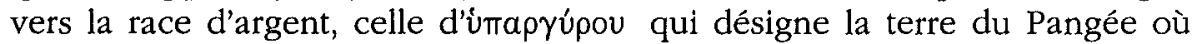
Rhésos sera caché. Sans doute le poète fait-il une allusion voulue à la diction hésiodique en utilisant ici cet adjectif pour qualifier la terre du Pangée qu'il

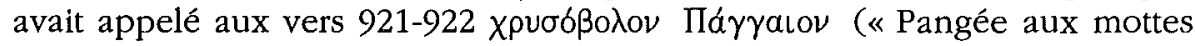
d'or »). L'appellation de daimôn et la demeure souterraine sont donc des caractéristiques des héros cultuels qui sont communes à Rhésos, et nous pouvons supposer que son statut après la mort est présenté comme celui d'un héros de ce type ${ }^{22}$.

Considérons maintenant la présence de Bacchos. Dans cette description du statut post mortem de Rhésos, les vers $972-973$ sont ceux qui ont posé le plus de difficultés aux éditeurs et aux commentateurs du texte. Les princi-

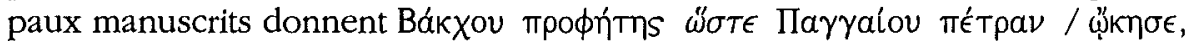

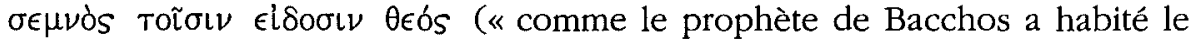
mont Pangée, dieu vénérable pour ceux qui savent »). Seul $\mathrm{Q}$, manuscrit que l'on peut qualifier de «marginal » par rapport aux deux grandes familles, ainsi qu'une correction du manuscrit $P$ donnent la leçon ö $\sigma \tau \epsilon$. En conséquence, la majorité des éditeurs a adopté la leçon $\ddot{\omega} \sigma \tau \epsilon$. Pour les commentateurs se posait alors le problème de savoir qui était ce prophète de Bacchos, auquel Rhésos était comparé. La question a souvent été débattue et plusieurs répon-

19 G. Nagy, The Best of the Achaeans. Concepts of the Hero in Arcbaic Greek Poetry, Baltimore/Londres, 1979, p. 154 (tr. fr. par J. Carlier et N. Loraux, Le Meilleur des Acbéens. La fabrique du béros dans la poésie grecque archaique, Paris, 1994, p. 192).

20 E. Rodhe, Psycbe: Seelencult und Unsterblicbkeitsglaube der Griecben, Fribourg, $1898^{2}$ [Fribourg, 1893-1894], I, p. 111-145 (tr. fr. par A. Reymond, Psycbé. Le culte de l'âme chez les Grecs et leur croyance à l'immortalité, Paris, 1928, p. 92-120).

21 Le $\theta$ vitol des manuscrits a été corrigé par Pepmüller en $\theta \nu \eta$ toís qui est adopté par Rzach et Mazon. Cette correction « est irrecevable parce qu'elle n'est pas grammaticale ", souligne M. Crubellier dans son article intitule "Le mythe comme discours. Le récit des cinq races humaines dans les Travaux et les Jours", in P. JUDET DE LA COMBE, F. Blaise, Ph. Rousseau (éds), Le métier du mythe. Lectures d'Hésiode, Lille, 1996, p. 457. C'est ce que pense M.L. West qui explique que cette correction serait sans parallèle dans

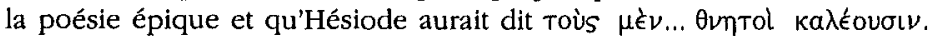

22 Concernant l'existence d'un culte héroïque de Rhésos, voir l'article de W. LEAF, "Rhesus of Thrace", JHS 35 (1915), p. 1-11, et, plus récemment, N. THeodossıev, "Monumental Tombs and Hero-Cults in Thrace during the 5th-3rd centuries B.C.", in V. PIRENNEDelforge, E. SuÁrez de la Torre (éds), Héros et béroines dans les mythes et les cultes grecs, Liège, 2000 (Kernos, suppl. 10), p. 435-447. 
ses ont été apportées : on y a vu Orphée (E. Maass ${ }^{23}, \mathrm{G}$. Murray $^{24}, \mathrm{~J}$. Rempe ${ }^{25}$, L. Weber ${ }^{26}$, etc.), Lycurgue (S. Musgrave ${ }^{27}$, F.A. Paley ${ }^{28}$, M.C. West ${ }^{29}$, etc.), Dionysos même (W. Ridgeway ${ }^{30}$ ) ou enfin, Zalmoxis. Cette dernière hypothèse est celle que soutient $A$. Nock ${ }^{31}$ contre celles citées précédemment. Le problème ne se pose pas s'il résulte, comme je le crois, d'un mauvais choix de texte. Ce qui est étrange tout d'abord, c'est que la Muse taise le nom de celui à qui elle compare Rhésos; on ne voit pas ce que pourrait signifier cet anonymat. Ensuite, sur le plan syntaxique, la combinaison du comparatif $\ddot{\sigma} \sigma \epsilon$ avec un verbe fini est assez inhabituelle dans la tragédie et nécessite un examen. Ceux qui choisissent de garder $\ddot{\sigma} \sigma \epsilon$ justifient cet emploi comme épique. Cependant, dans un article consacré à ces vers du $R b e ́ s o s^{32}$, J. Diggle démon-

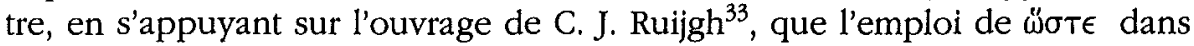
ces vers du Rhésos n'est pas conforme à l'emploi épique : dans les exemples que l'on trouve chez Homère, mais aussi dans les Hymnes et chez Hésiode,

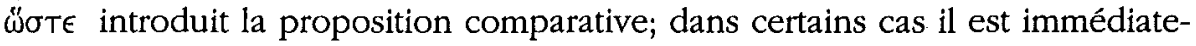
ment précédé par le sujet de la proposition principale (une fois par le sujet et le verbe, une autre fois par le sujet et le complément d'objet) et dans tous les cas, la proposition principale est résumée, après la comparaison, avec üs. Dans la tragédie, on rencontre quelques exemples de cet emploi de $̋ \sigma \tau \epsilon$, essentiellement dans des passages lyriques où l'on voit souvent l'affinité avec l'usage épique. Deux exemples se présentent dans des passages iambiques et pourraient donc offrir un parallèle à notre passage : il s'agit du fragment 840 Pearson [Radt] de Sophocle ${ }^{34}$ et des vers 1066-1067 des Bacchantes ${ }^{35}$. Dans les deux cas la syntaxe est difficile à comprendre. Cependant, là encore - du moins, dans les Bacchantes, puisqu'on ignore le contexte du fragment - la comparaison fait référence à un fait permanent, ce qui n'est pas le cas dans les vers du Rbésos où $\omega \sigma \sigma \tau \epsilon$ introduit un événement temporaire, qui a eu lieu

23 O.c. (n. 7), p. 66-71, 134-139.

24 G. Murray, Euripidis fabulae, Oxford, 1902-1909 (vol. III, 1913).

25 J. REMPE, De Rheso Thracum beroe, Diss. Münster, 1927, p. 26 sq.

26 L. WeBER, "Orpheus", RhM 81 (1932), p. 8.

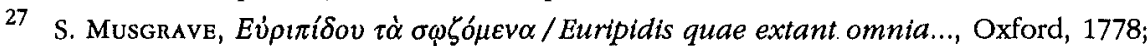
Fragmenta tragoediarum deperditarum, Glasgow, 1797.

28 F.A. PALEY, Euripides, Londres, 1857.

29 M.C. West, "The Lycurgus Trilogy", BICS 30 (1983), p. 63-64; ID., Studies in Aeschylus, Stuttgart, 1990, p. 26-32.

30 W. RIDGEWAY, "Euripides in Macedon", CQ 20 (1926), p. 17 sq.

31 A. Nock, "The End of the Rhesus", $C R 40$ (1926), p. 184-186.

32 J. Diggle, "The prophet of Bacchus: Rhesus 970-973", SIFC 5 (1987), p. 167-172. Repris dans: Euripidea. Collected Essays, Oxford, 1994, p. 320-326.

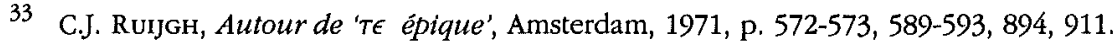

34 Cf. J.D. Denniston, The Greek Particules, Oxford, $1954^{2}$ [Oxford, 1934], p. 588; o.c. (n. 33), p. 994.

35 O.c. (n. 33), p. 997-998. 
une fois pour toutes ${ }^{36}$. Non seulement l'emploi de $\ddot{\sigma \tau \epsilon}$ dans ces vers du Rbésos s'écarte de l'emploi qui en est fait ailleurs mais de plus, il ne donne pas à la phrase un sens satisfaisant : le "prophète de Bacchos " est laissé dans l'anonymat et il est qualifié successivement de «prophète et de dieu vénérable », ce qui paraît étrange. C'est pourquoi, comme J. Diggle, je choisis de rejeter la leçon $\omega ̈ \sigma \tau \epsilon$. Cependant, si J. Diggle pense que le relatif et non le

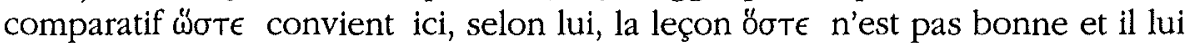
préfère la correction ös $\gamma \epsilon$ de A.H. Matthiae ${ }^{37}$ (qui est également suivie par J. Zanetto ${ }^{38}$ ). En effet, A.H. Matthiae, comme J. Diggle, met en avant le fait

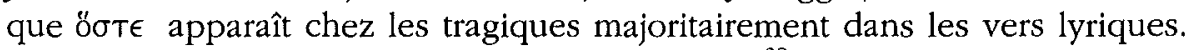
Pour ma part, je pense, comme U. v. Wilamowitz ${ }^{39}$, que l'on peut considérer öøte comme un archaïsme délibéré, et c'est pourquoi je choisis de le garder. Un homérisme me semble en accord avec le travail de réécriture d'Homère auquel se livre le poète du Rbésos dans ce passage et dans toute la pièce. Le

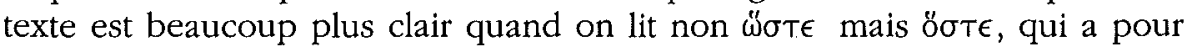

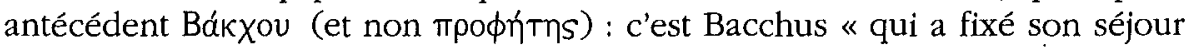
sur le mont Pangée » et qui est « un dieu vénérable pour ceux savent », et non le prophète, comme dans la lecture avec $\ddot{\sigma} \sigma \epsilon$. Notre lecture trouve appui dans le vers 1267 d'Hécube, cité par J. Diggle : «Dionysos rend des oracles aux Thraces, il l'a prédit " et l'existence d'un oracle de Dionysos sur le mont Pangée est confirmée par Hérodote (VII, 111), également cité par J.

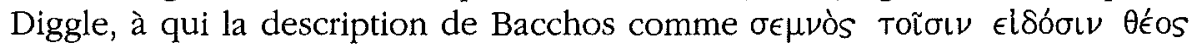
semble appropriée, en regard de ce qui est dit dans les Bacchantes, 72-73:

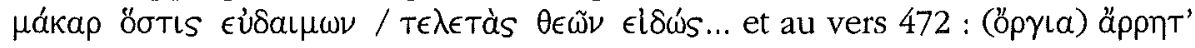

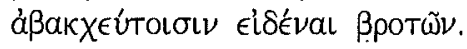

Rhésos devient donc «prophète de Bacchos " ${ }^{40}$. Nous ignorons la signification exacte de cette expression mais, comme nous l'avons vu, Bacchos avait un oracle, et nous pouvons supposer que Rhésos devient interprète des oracles du dieu auprès des hommes. La question se pose de savoir pourquoi Rhésos devient le serviteur de ce dieu précisément. Si ce n'est pas Bacchos qui a tiré Rhésos des Enfers, un des mythes relatifs au dieu cependant, le mythe orphique qui en faisait un dieu chthonien de la mort et de la régénération et auquel les mystères se référaient, en faisait le fils de Perséphone. Les deux divinités sont donc liées. Bacchos entre dans ce réseau de liens que la Muse a tissés entre les divinités qui avaient des mystères et appartenaient toutes à la sphère mystique. Cependant, remarquons que la Muse n'indique

36 O.c. (n. 33), p. 998.

37 A.H. Matthiae, Euripidis Tragoediae, Leipzig, 1824.

38 J. ZANETTO, Euripides Rhesus, Stuttgart/Leipzig , 1993.

39 U. von Wilamowitz-Moellendorff, "Lesefrüchte", Hermes 61 (1926), p. 284-289. Repris dans: Kleine Schriften IV, Berlin, 1962, p. 411-416, p. 285, n. 1; ID., Der Glaube der Hellenen II, Darmstadt, 1931-1932, p. 74, n. 2.

40 Baccbos, «le Bacchant », désigne à la fois les fidèles de Dionysos et le dieu comme inspirateur de leur état de transe, en tant que dieu des pratiques orgiaques. 
pas les liens existant entre Bacchos et Perséphone, et que le rôle joué ici par les deux divinités n'est pas le même que celui dont témoignent par exemple les lamelles de Pélinna ${ }^{41}$. Rien ne nous permet ici de reconstituer une eschatologie de type orphico-dionysiaque.

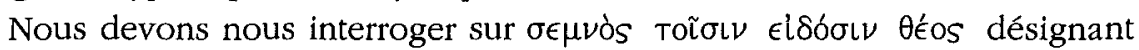
Bacchos. En effet, le Toĩ pour qui Dionysos serait vénérable alors qu'en fait Dionysos, en tant que dieu du panthéon grec aussi ancien que les autres; était honoré de tous les Grecs. Ce que signifie alors l'expression, c'est que le commun des mortels ne sait pas à quel point Dionysos est $\sigma \epsilon \mu \nu o ́ s$, ne connaît pas réellement la divinité dans toute sa puissance. C'est ce qui se passe dans les Bacchantes où Penthée se refuse à connaître vraiment ce qu'est Dionysos, comme les ménades du chœur le connaissent. Ces dernières utilisent les mêmes termes que dans le Rhésos pour désigner ceux qui savent voir le dieu, qui sont initiés à ses mystères ( $c f$. les vers $72-73$ et 472 des Bacchantes cités plus haut).

Les liens que le texte du Rbésos établit entre ces réalités mystiques que sont Orphée, les mystères, Perséphone, la vie après la mort et Bacchos nous posent problème. Or, le poète du Rhésos ne les explicite pas et, s'il nous semble pouvoir reconnaître des éléments « orphiques ", il nous est impossible de reconstruire un tout cohérent. Cela revient en effet à vouloir éclairer une réalité pour le moins obscure, l'orphisme, par un texte qui ne l'est pas moins. Car le matériau orphique n'est pas le seul à être utilisé par le poète qui, comme nous l'avons vu, emprunte également à Hésiode.

S'il est impossible d'identifier dans la description du statut de Rhésos après la mort une doctrine mystique et si ce statut apparaît plutôt comme une énigme que le poète du Rbésos construit à partir d'éléments appartenant à des tradition hétérogènes, c'est que cet état n'est pas un état défini mais une somme de contradictions. La Muse commence par dire au vers 962 que

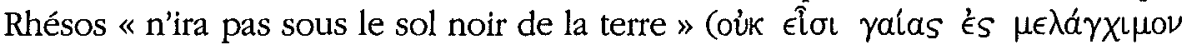
$\pi \in ́ \delta 0 \nu$ ) et qu'elle va inciter Perséphone à faire remonter l'âme de Rhésos des Enfers, ce qui laisse à penser que Rhésos va bénéficier de l'immortalité. Pourtant, - et c'est là une première contradiction, - la Muse dit ensuite que Rhésos'sera pour elle « comme mort et ne voyant plus la lumière pour le reste

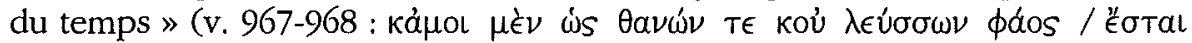

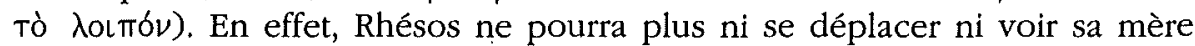
(v. 968-969), parce qu'il sera « caché dans les antres de la terre » (v. 970 :

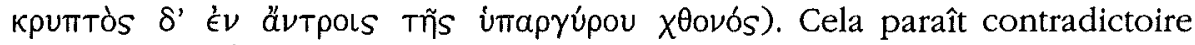
avec le vers 962 : Rhésos, s'il ne sera pas aux Enfers, demeurera quand même

41 Cf. F. GraF, "Dionysian and Orphic Eschatology: New Texts and Old Questions", in Th. Carpenter, Ch. Faraone (éds), Masks of Dionysus, Ithaca/Londres, 1993, p. 239-258 (p. 243 sq.). Voir aussi C. Calame, "Invocations et commentaires « orphiques »: transpositions funéraires et discours religieux", in M.M. MaCtoux, E. Geny (éds), Discours religieux dans l'Antiquité, Besançon/Paris, 1996, p. 11-30. 
sous la terre. Le vers 971 résume le futur état de Rhésos : $\alpha \nu \theta \rho \omega \pi$ o $\delta \alpha l \mu \omega \nu$

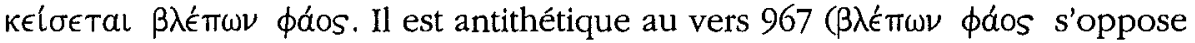

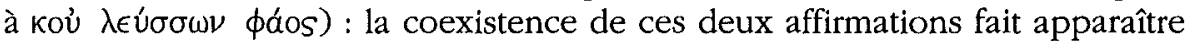

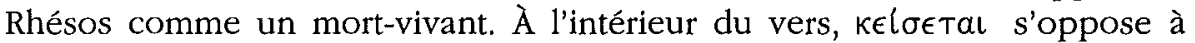

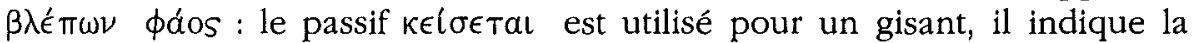

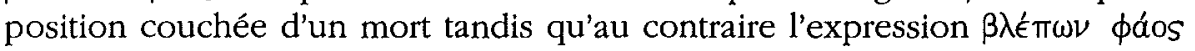

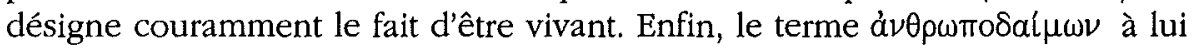
seul est contradictoire: plutôt que de le comprendre comme signifiant " homme devenu daimôn », il faut le comprendre comme marqueur d'une double nature, celle d'" homme-démon », autrement dit d'un être marqué à la fois par la mortalité et l'immortalité. Ainsi, l'état de Rhésos est un état de tensions, intermédiaire, indéfinissable, où la mortalité est concomitante à l'immortalité.

Cet état n'est ni l'immortalité telle que la concevaient les mystiques ni celle du héros épique. En effet, dans l'épopée homérique, il n'est pas question de la survie cultuelle du héros, qui est à la frontière de l'épopée. En revanche, l'immortalité du héros, chez Homère, passe par le chant et suppose une claire distinction entre le monde des vivants et le monde des morts. La Muse ne peut chanter Rhésos comme un héros épique puisque, tout en étant mort, il ne sera pas dans le monde des morts ${ }^{42}$. Il ne jouira pas non plus de la félicité éternelle dans l'île des Bienheureux aux confins du monde des vivants, puisqu'il sera caché là, sous la terre du Pangée. La tragédie, en tant que genre, n'ignore pas le culte avec lequel elle a partie liée, ni les croyances en une vie après la mort, comme le fait l'épopée qui les relègue dans ses marges, mais elle les intègre, tout en s'en séparant. La Muse ne tient pas un discours orphique; elle n'affirme pas une croyance en une vie meilleure post mortem et ne considère pas la mort comme un accomplissement. Pour elle, Rhésos sera comme mort le reste du temps (v. 962), et c'est la raison pour laquelle elle le pleure et s'apprête à porter son deuil, comme on le voit dans les vers 974-982. La description de l'immortalité de Rhésos et de sa position de prophète de Bacchos est encadrée par l'affirmation de sa mort. Dire la mort, c'est ce que ne cesse de faire la Tragédie, et le Rbésos, contrairement à ce que l'on pourrait penser au premier abord, ne dit pas autre chose. La Muse tient un discours tragique. À partir du moment où son fils est mort, elle ne peut plus être la Muse telle que la concevaient Homère et Hésiode, qui chantait dans les banquets des dieux pour réjouir leur coeur, qui faisait oublier aux hommes leurs soucis en. leur chantant les exploits des héros. En faisant de la Muse épique une mère et en la plaçant au cœur de l'action, le poète du Rbésos la transforme : par le biais de cette dramatisation de la Muse, il réfléchit sur l'épopée et sur le héros épique. Le roi thrace qui apparaît dans le chant X de l'Iliade est en fait un héros tragique : voilà ce que le Rbésos met en évidence. Les termes de la Muse décrivant le futur état de son fils sont parfaitement adaptés à son statut poétique: Rhésos apparaît bien dans la 
tragédie qui porte son nom comme un être fantomatique, un mort-vivant qui ne vient sur scène que pour mourir, sans avoir le temps d'agir ni même d'avoir conscience de sa situation. Il est une apparition lumineuse, l'image du kléos mais sans efficience. Rhésos n'est qu'un être de langage ${ }^{43}$ et non un être d'action. C'est cela qui le distingue des autres héros tragiques de la pièce. Hector quant à lui est tragique dans ce sens que, du début à la fin de la pièce, il est aveugle à ce qui arrive : il ne comprend pas le rôle fondamental qu'aurait pu jouer Rhésos et ne saisit pas que lorsque Rhésos est assassiné, il perd celui qui aurait pu sauver Troie. Hector fait peu de cas de la venue de Rhésos comme il fait peu de cas de sa mort. Mais, si Hector, comme Rhésos, n'a pas conscience de ce qui lui arrive, lui est dans l'action; il a tout le temps d'agir. Rhésos au contraire est un héros tragique particulier parce que la possibilité d'agir ne lui est pas donnée.

Sur le plan de cette réflexion méta-poétique, la présence de Dionysos, le dieu de la Tragédie, n'est peut-être pas sans signification. Le fait que, par sa mort, Rhésos devienne «prophète de Bacchos » signifierait qu'il devient héros tragique.

Université Charles de Gaulle, Lille III

Caroline PLICHON

Savoirs et Textes, U.M.R 8519.

43 On peut rapprocher son nom - 'Pñoos - du terme p̀ñoıs qui désigne la parole. 